\title{
Gestión de la Calidad desde la ISO 9001: Análisis Teórico de Casos
}

\section{Quality Management since the ISO 9001: Theoretical Case Analysis}

DOI: http://dx.doi.org/10.17981/cultedusoc.8.1.2017.03

Leilys Zullin Ortega Loaiza ${ }^{1}$, Karen Inés Almanza Vides² y Norelys Genoveba Cárdenas Fragozo ${ }^{3}$

Universidad de La Guajira

\begin{abstract}
Resumen
El presente artículo se orienta al análisis sistemático que tienen los procesos de gestión de la calidad desde la ISO-9001, especialmente en el desarrollo organizacional, productividad y competitividad de las empresas. Para tal efecto se adelantó una investigación de corte documental, enriquecida con la revisión sistemática de casos y artículos publicados en revistas especializadas. Dentro de los resultados derivados de la revisión sistemática, se identificó que los procesos de apropiación social de la ISO-9001 inciden positivamente en la gestión de la calidad de las empresas, así como en el cumplimiento de los estándares que fortalecen su nivel de formalización, reconocimiento y promueven la implementación de estrategias de desarrollo organizacional para el logro de sus objetivos corporativos y misionales.
\end{abstract}

Palabras clave:Gestión de calidad, ISO-9001, desarrollo organizacional, productividad, competitividad.

Recibido: Agosto 03 de 2016 Aceptado: Noviembre 15 de 2016

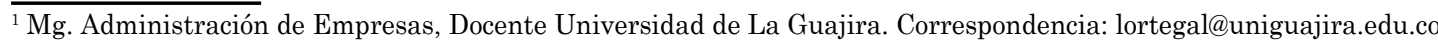

${ }^{2}$ PhD. Ciencias Políticas, Docente Universidad de La Guajira, Correspondencia: kalmanzav@uniguajira.edu.co

${ }^{3}$ Ph.D. en Ciencia Mención Gerencia. Docente Universidad de La Guajira. Correspondencia: ncardenas@uniguajira.edu.co
} 


\begin{abstract}
This paper is oriented to the systematic analysis of quality management processes from the NTC-ISO 9001, especially in in the organizational development, productivity and competitiveness of companies. For this purpose, a documentary investigation was carried out, enriched with the systematic review of cases and articles published in specialized journals. Among the results derived from the systematic review, it was identified that the processes of social appropriation of the NTC-ISO 9001 have a positive impact on the quality management of small and medium-sized companies, as well as compliance with standards that strengthen their level of formalization, recognition and promote the implementation of organizational development strategies for the achievement of corporate and mission objectives.
\end{abstract}

Keywords: Quality Management, ISO-9001, organizational development, productivity, competitiveness.

\title{
Introducción
}

Los procesos de gestión de la calidad comprenden un escenario de alto impacto para los procesos de desarrollo organizacional y por ende indicen significativamente en los niveles de formalización, estandarización de procesos, productividad y competitividad de las empresas. Este artículo, ha sido derivado de un proceso de revisión sistemática y reflexión teórica sobre diferentes análisis de casos y experiencias implementadas con la ISO-9001 y su incidencia en los procesos de gestión de la calidad.

Dentro de las diferentes definiciones sobre gestión de la calidad, la norma enmarca este tópico en términos generales, la "Gestión de la Calidad comprende el conjunto de acciones, planificadas y sistemáticas, que son necesarias para proporcionar la confianza adecuada de que un producto o servicio va a satisfacer los requisitos dados sobre la calidad" (ISO-9001, 2013, online).

Dentro de los autores clásicos, Feigenhaum (1994) define la Gestión de Calidad como "el conjunto de las características del producto de marketing, ingeniería, fabricación y mantenimiento a través del cual el producto satisface las expectativas del cliente" (p. 274).

Por su parte, Rey (2005) plantea que la "Gestión de calidad tiene impacto estratégico en la empresa y representa una oportunidad competitiva, poniendo especial énfasis en el mercado y las necesidades del cliente" (p. 7).

Uno de los aspectos que ha tomado mayor vigencia, comprende el desarrollo de sistemas de gestión de la calidad sustentada en los procesos e interacciones de la organización, lo cual es denominado por algunos autores como Yáñez (2008) el "enfoque basado en procesos".

Complementariamente, López (2006) considera que la gestión de un sistema de calidad comprende "la estructura organizativa, las responsabilidades, los procesos y los recursos necesarios para llevar a cabo la gestión de la calidad. Se aplica en todas las actividades realizadas en una empresa y afecta a todas las fases, desde el estudio de las necesidades del consumidor hasta el servicio postventa" (p. 12).

Desde la perspectiva de López (2006) se resalta la importancia de la gestión de calidad, bajo el escenario de la implementación de Sistemas de Control de la Gestión de Calidad, donde se articulan la gestión de calidad, el sistema de control de la gestión y la opinión de los clientes, tal como lo ilustra en el siguiente diagrama. 


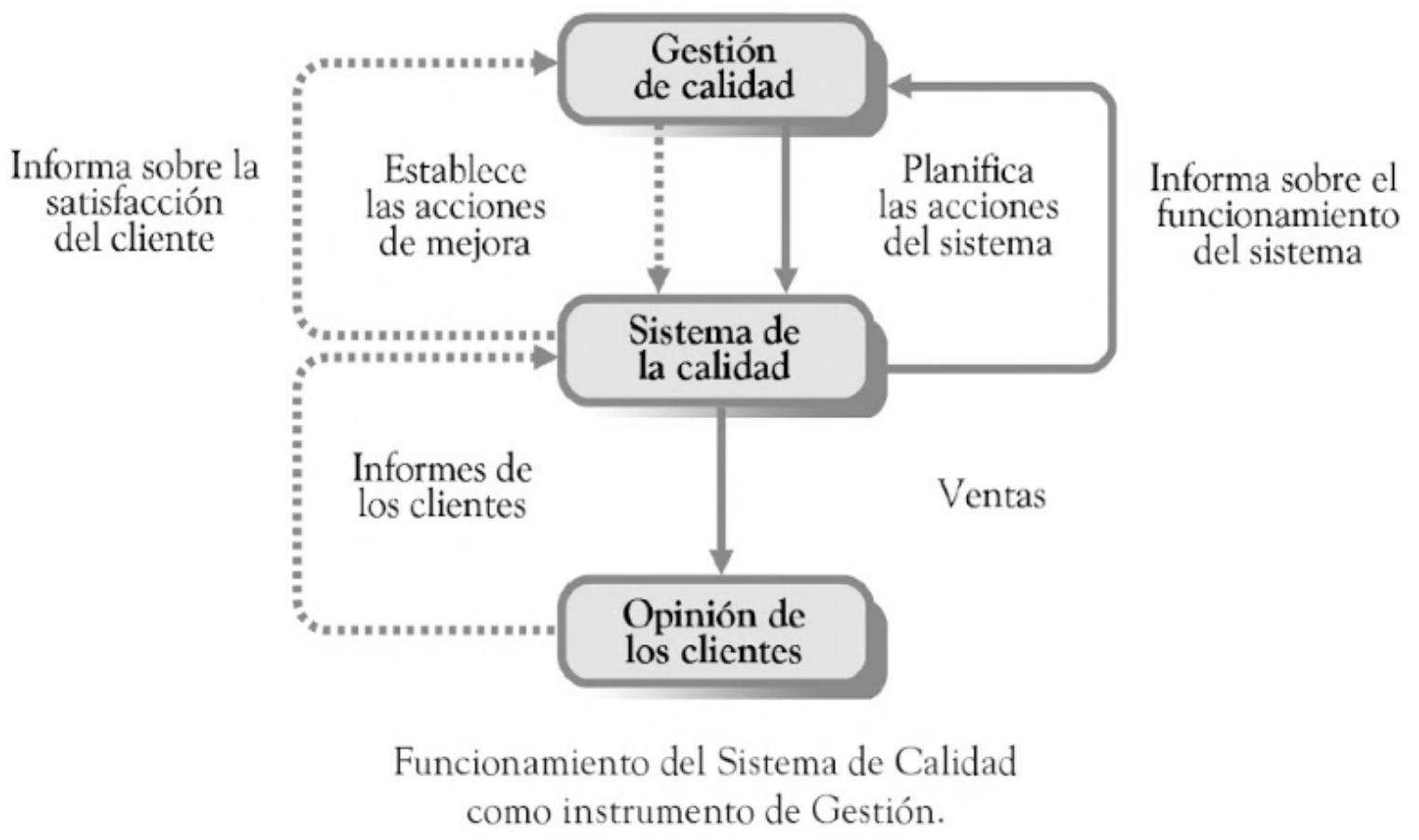

Figura 1. Funcionamiento del Sistema de Calidad como Instrumento de Gestión Fuente: López (2016, p. 13)

Tal como se argumenta en la NTC-ISO 9001 en el análisis de la gestión de calidad desde el enfoque de procesos se considera que:

Aunque existen multitud de definiciones para este término, podría definirse como organización de personas, procedimientos y medios que, en trabajo coordinado, son necesarios para lograr un final concreto. Constituyen una secuencia repetitiva de entradas y salidas, medibles y con incorporación de un valor añadido. Dependiendo de su amplitud y complejidad puede dividirse en subprocesos y/o agruparse en macroprocesos (ISO-9001, 2013, online).

Por su parte, Vinuesa (2013) resalta el proceso de Gestión de Calidad desde la ISO-9001, bajo los siguientes términos:

De acuerdo con la norma ISO-9001:2000, la organización debe planificar e implementar los procesos de seguimiento, medición, análisis y mejora necesarios para demostrar la conformidad del producto, asegurar la conformidad de los procesos y mejorar continuamente la eficacia del sistema de gestión de la calidad. En estos procesos de control, y consecuentemente de medición, no puede obviarse el uso de indicadores no financieros (p. 99).

Dentro de los diferentes estudios sobre el tema de la Gestión de Calidad, cabe resaltar el análisis bibliométrico de estudios publicados en revistas hispanoamericanas, por Ruiz-Torres, Ayala-Cruz, Alomoto y Acero Chavez (2015), a partir del cual los autores afirman que: 
La revisión ha propuesto áreas de interés para los investigadores y, al mismo tiempo, temas de oportunidad. En la última década varios estudios en Estados Unidos y Europa han considerado las proposiciones de empresas para integrar y lograr un mejor balance entre iniciativas de mejora de la calidad y la gestión de innovación. Aunque se desprende que hay un gran interés en el tema, las investigaciones se enfocan más hacia el impacto de la calidad en el desarrollo de nuevos productos y procesos, y no a temas como procesos de difusión en la región o iniciativas para fomentar la adopción y la institucionalización de la innovación como un elemento cultural en las empresas (p. 328).

Los conceptos anteriormente citados, permiten comprender la importancia que tiene el sistema de gestión de la calidad para las organizaciones y especialmente frente al desarrollo organizacional, la productividad y competitividad de las empresas. A continuación se formulan los aspectos metodológicos y resultados derivados del proceso de revisión sistemática de los casos seleccionados.

\section{Método}

La aproximación metodológica del presente estudio comprende una investigación de corte documental, con la técnica de revisión sistemática de artículos publicados en revistas especializadas. Para tal efecto se contó con una muestra de artículos referentes al abordaje del objeto de estudio sobre la incidencia de la ISO-9001 en el contexto de los procesos de desarrollo organizacional. A nivel procedimental, los artículos seleccionados fueron clasificados en función de su pertinencia y relevancia e incorporados en las tablas de resumen analítico que se presentan a continuación en los resultados.

\section{Resultados}

A continuación se presentan los resultados derivados de la revisión sistemática de los casos contemplados en los artículos referentes a la incidencia de la incorporación de la ISO-9001. En primera instancia se presenta la siguiente tabla de resumen sistemático que contiene los principales elementos de análisis teórico sobre los estudios seleccionados.

Tabla 1

Revisión Sistemática: Estudios de ISO-9001

\begin{tabular}{|c|c|c|}
\hline $\begin{array}{l}\text { Autores } \\
\text { (año) }\end{array}$ & Título & Resumen Analítico \\
\hline $\begin{array}{l}\text { Carmona- } \\
\text { Calvo, } \\
\text { Suárez, } \\
\text { Calvo-Mora } \\
\text { \& Periáñez- } \\
\text { Cristóbal } \\
(2016)\end{array}$ & $\begin{array}{l}\text { Sistemas de gestión } \\
\text { de la calidad: un } \\
\text { estudio en empresas } \\
\text { del sur de España y } \\
\text { norte de Marruecos. }\end{array}$ & $\begin{array}{l}\text { En el trabajo se analizan los niveles de implantación de } \\
\text { sistemas de gestión de la calidad (SGC) con } 322 \text { empresas } \\
\text { del sur de España y norte de Marruecos, y las motivaciones, } \\
\text { beneficios o barreras detectadas. En los dos entornos se } \\
\text { comparten algunas dificultades propias tales como el costo } \\
\text { de implementación, la falta de experticia y tiempo real } \\
\text { para el desarrollo de los procesos, sin embargo también se } \\
\text { presentan diferencias referentes al logro de los estándares de } \\
\text { certificación de la calidad y el nivel de estandarización de los } \\
\text { procesos (p.8). }\end{array}$ \\
\hline
\end{tabular}




$\begin{array}{lll}\begin{array}{c}\text { Autores } \\ (\mathrm{anno})\end{array} & \text { Título } & \text { Resumen Analítico }\end{array}$

Bonilla

García

(2010)

Heras, Marimon \& Casades (2009)

Vinuesa \& Zuera (2010)

Villalbí, Ballestín, Casas \& Subirana (2012)
Esta investigación compara la calidad de los sistemas de gestión de recursos humanos entre las empresas que cuentan o no con la certificación ISO 9001:2000. Una herramienta basada en los ocho principios de los sistemas de gestión de la calidad, permitió que las organizaciones los evaluaran y describieran las evidencias/indicadores asociados, determinando su impacto. Los resultados indican que no existen diferencias entre los grupos de empresas con respecto a la presencia e impacto de las evidencias/indicadores. Se identifica la necesidad de concebir programas de certificación específicos para RR.HH., así como de planes y estrategias orientados a la mejora continua y relaciones con el proveedor, así como de desarrollar prácticas asociadas a indicadores para el seguimiento y control de la gestión organizacional (p. 39).

En este artículo se evalúa el impacto de las herramientas para la gestión de la calidad en la competitividad de las organizaciones, tomándose como referencia dos modelos distintos de gestión de la calidad: el establecido por el estándar ISO 9001:2000 y el del modelo de autoevaluación EFQM. A partir de los resultados se evidencia que las empresas que incorporan la norma, desarrollan procesos de mejoramiento continuo que incrementan significativamente sus niveles de productividad y competitividad (p. 7).
La certificación ISO 9000 en el sector industrial del mueble: evidencias sobre la cultura de calidad total y las ventajas que la caracterizan
Este estudio analiza si el nivel de implantación de prácticas de gestión de la calidad total, y el nivel de resultados empresariales, son factores que caracterizan a las empresas certificadas con la norma ISO 9000 en la industria nacional del mueble. Asimismo, se comprueba la posible relación de determinados aspectos TQM con la mejora de los resultados empresariales (p. 77).

Se presenta la experiencia de introducción de acciones de mejora de la calidad en una organización de salud pública. Tras la acreditación ISO 17025 del laboratorio de control oficial obtenida por requisito legal, la dirección acordó en 2003 extender la política de calidad, poniendo en marcha un repertorio de acciones mediante el análisis de procesos y la formulación de acciones de mejora, la mayor formalización de procedimientos, el conocimiento de la opinión de los clientes, la creación de grupos de mejora, y la auditoría o certificación externa. La organización respondió de forma diversa a estas iniciativas. Someter los servicios a auditoría o certificación externa parece el motor más poderoso para el cambio. El estudio de costes de la actividad permite valorar que, en 2010 , el $75 \%$ del gasto total se realiza en servicios de salud pública sujetos a auditoría/certificación externa (p. 379). 


\begin{tabular}{|c|c|c|}
\hline $\begin{array}{l}\text { Autores } \\
\text { (año) }\end{array}$ & Título & Resumen Analítico \\
\hline $\begin{array}{l}\text { Mena, } \\
\text { Martínez, } \\
\text { Púa, Suñé, } \\
\text { Jané, \& Sala } \\
(2007)\end{array}$ & $\begin{array}{l}\text { Descripción } \\
\text { del proceso de } \\
\text { certificación ISO } \\
\text { 9001/2000 en el } \\
\text { área de nutrición } \\
\text { parenteral }\end{array}$ & $\begin{array}{l}\text { Este trabajo describe el proceso de implantación de la norma } \\
\text { UNE-EN-ISO-9001/2000 en el área de nutrición parenteral, } \\
\text { desarrollado con el propósito de analizar el aseguramiento } \\
\text { de la calidad y seguridad e incrementar la satisfacción del } \\
\text { usuario ha llevado a organizaciones del ámbito sanitario a } \\
\text { integrar un sistema de gestión de calidad estructural (p. } \\
\text { 370). }\end{array}$ \\
\hline $\begin{array}{l}\text { Espín, } \\
\text { Jiménez \& } \\
\text { Costa } \\
(2014)\end{array}$ & $\begin{array}{l}\text { La gestión de calidad: } \\
\text { importancia de la } \\
\text { cultura organizativa } \\
\text { para el desarrollo de } \\
\text { variables intangibles }\end{array}$ & $\begin{array}{l}\text { El objetivo del estudio consiste en analizar cómo las } \\
\text { empresas con una mayor orientación hacia la calidad } \\
\text { promueven el desarrollo de determinadas prácticas } \\
\text { organizativas de carácter intangible que apoyen la obtención } \\
\text { de beneficios. Algunas de estas variables determinantes de } \\
\text { la orientación a la calidad son la cultura organizativa, la } \\
\text { orientación al mercado, la gestión de recursos humanos y el } \\
\text { liderazgo (p. 115). }\end{array}$ \\
\hline $\begin{array}{l}\text { Carballido, } \\
\text { Manuel \& } \\
\text { Rivas } \\
(2008)\end{array}$ & $\begin{array}{l}\text { Desempeño de las } \\
\text { organizaciones } \\
\text { mexicanas } \\
\text { certificadas en la } \\
\text { norma ISO 9001: } \\
2000\end{array}$ & $\begin{array}{l}\text { Esta investigación describe la situación de la certificación } \\
\text { en México en la norma ISO 9001:2000, se demuestra que el } \\
\text { desempeño de una organización sí mejora cuando se implanta } \\
\text { un programa ISO 9001:2000, siempre y cuando exista una } \\
\text { motivación activa (p. 107). }\end{array}$ \\
\hline $\begin{array}{l}\text { León, \& } \\
\text { Valenzuela } \\
\text { (2014) }\end{array}$ & $\begin{array}{l}\text { Aprendizaje, } \\
\text { innovación y gestión } \\
\text { tecnológica en la } \\
\text { pequeña empresa: } \\
\text { Un estudio de } \\
\text { las industrias } \\
\text { metalmecánica y } \\
\text { de tecnologías de } \\
\text { información en } \\
\text { Sonora. }\end{array}$ & $\begin{array}{l}\text { Este trabajo plantea resultados de un estudio empírico sobre } \\
\text { las micro, pequeña y mediana empresas de los sectores } \\
\text { mencionados, permitiendo conjeturar una correlación directa } \\
\text { entre los niveles de capacidades acumuladas y de gestión } \\
\text { tecnológica, frente a los procesos de gestión de la calidad (p. } \\
\text { 253). }\end{array}$ \\
\hline
\end{tabular}

Fuente: Elaboración propia

\section{Discusión}

Los estudios analizados en la revisión sistemática, permiten evidenciar el impacto significativo que tiene la incorporación de la ISO-9001 en los niveles de desarrollo organizacional y productividad y competitividad de las empresas. Cabe resaltar la importancia que tienen los sistemas de gestión de la calidad en la definición, estandarización y optimización de los procesos de la organización, reduciendo los trámites, tiempos y costes.

En la medida en que las empresas incorporan las normas técnicas como es el caso de la ISO-9001, se observa que los procesos de desarrollo organizacional inciden a su vez en el mejoramiento continuo y el aseguramiento integral de la calidad. Este proceso de mejoramiento continuo, implica adicionalmente que la organización debe asumir una perspectiva de monitoreo y asistencia técnica a nivel multidimensional con un sistema de retroalimentación integral para el control y la gestión de calidad. 
Los procesos de gestión de la calidad no son automáticos y por lo tanto el desarrollo permanente del potencial talento humano vinculado juega un papel relevante para la organización. En este sentido se identifica una relación estrecha entre la gestión de la calidad basada en procesos y el desarrollo de las capacidades dinámicas, sustentadas en el aprendizaje organizacional.

\section{Referencias}

Bonilla García, J. (2010). La calidad de los sistemas de gestión de recursos humanos en función de la tenencia o no de la certificación ISO 9001: 2000: algunas evidencias/indicadores asociados. Estudios Gerenciales, 26(115), 39-64.

Carballido, N., Manuel, V., \& Rivas Tovar, L. A. (2008). Desempeño de las organizaciones mexicanas certificadas en la norma ISO 9001: 2000. Estudios Gerenciales, 24(108), 107-128.

Carmona-Calvo, M. A., Suárez, E. M., Calvo-Mora, A., \& Periáñez-Cristóbal, R. (2016). Sistemas de gestión de la calidad: un estudio en empresas del sur de España y norte de Marruecos. European Research on Management and Business Economics, 22(1), 8-16.

Espín, J. A. G., Jiménez, D. J., \& Costa, M. M. (2014). La gestión de calidad: importancia de la cultura organizativa para el desarrollo de variables intangibles. $R e$ vista Europea de Dirección y Economía de la Empresa, 23(3), 115-126.

Feigenbaum, A. (1994). Control Total de la Calidad. México: Continental.

Heras, I., Marimon, F., \& Casades, M. (2009). Impacto competitivo de las herramientas para la gestiøn de la calidad. Cuadernos de Economía y Dirección de la Empresa, 12(41), 7-35.

ISO 9001 (2013). ISO 9001 calidad. Sistemas de Gestión de Calidad. Disponible en: http://iso9001calidad.com/

León, J. G. M., \& Valenzuela, A. V. (2014). Aprendizaje, innovación y gestión tecnológica en la pequeña empresa: Un estudio de las industrias metalmecánica y de tecnologías de información en Sonora. Contaduría y administración, 59(4), 253-284.

Mena, M. M., Martínez, S. F., Púa, Y. L., Suñé, E. L., Jané, C. C., \& Sala, J. R. (2007). Descripción del proceso de certificación ISO 9001/2000 en el área de nutrición parenteral. Farmacia Hospitalaria, 31(6), 370-374.

Rey, S. L. (2005). Implantación de un sistema de calidad: los diferentes sistemas de calidad existentes en la organización. Santiago de Vigo: Ideaspropias Editorial SL.

Ruiz, L. (2004). La calidad por calidad total en la empresa Moderna. Madrid: Alfaomega Grupo Editores S.A.

Ruiz-Torres, A. J., Ayala-Cruz, J., Alomoto, N., \& Acero-Chavez, J. L. (2015). Revisión de la literatura sobre gestión de la calidad: caso de las revistas publicadas en Hispanoamérica y España. Estudios Gerenciales, 31(136), 319-334.

Villalbí, J. R., Ballestín, M., Casas, C., \& Subirana, T. (2012). Gestión de calidad en una organización de salud pública. Gaceta Sanitaria, 26(4), 379-382. 
Vinuesa, L. M. M. (2013). Gestión de la Calidad Total e indicadores no financieros: reflejo del valor de la certificación ISO 9001: 2000. Revista Europea de Dirección y Economía de la Empresa, 22(2), 97-106.

Vinuesa, L. M., \& Zuera, J. G. (2010). La certificación ISO 9000 en el sector industrial del mueble: evidencias sobre la cultura de calidad total y las ventajas que la caracterizan. Investigaciones Europeas de Dirección y Economía de la Empresa, 16(1), 77-101.

Yáñez, C. (2008). Sistema de gestión de calidad en base a la norma ISO 9001. Recuperado de: http://internacionaleventos.com/articulos/articuloISO.pdf. 